\title{
Ethical implications of heart transplantation in elderly patients
}

Robert C. Robbins, MD

From the Department of Cardiothoracic Surgery, Stanford University School of Medicine, Falk Cardiovascular Research Center, Stanford, Calif.

Received for publication Nov 21, 2000; accepted for publication Nov 29, 2000.

J Thorac Cardiovasc Surg 2003;125:S62-3 Originally published in J Thorac Cardiovasc Surg 2001;121:434-5.

Copyright $\left({ }^{\circ} 2003\right.$ by The American Association for Thoracic Surgery

$0022-5223 / 2003 \$ 30.00+0$

doi: $10.1067 / \mathrm{mtc} .2003 .227$

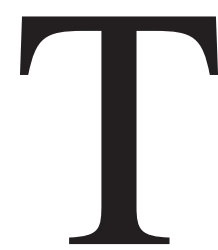

he results of cardiac transplantation in patients over 69 years of age reported by Blanche and associates [J Thorac Cardiovasc Surg 2001;121:532-41] demonstrate that short-term survival can be achieved in a very select, small group of such patients. As the authors have stated, the statistical comparison of this small group of older patients to younger recipients is problematic because the selection process was not uniform for both groups of patients. These data do not justify the elimination of older age as a selection criterion for cardiac transplantation, because most reports identify increased recipient age as a risk factor for post-transplantation mortality. Expanding the upper age limit will result in a further distortion of the current recipient demand/donor supply dilemma and will produce an increased mortality for all patients awaiting cardiac transplantation. The article by Blanche's group does raise many important moral, ethical, and practical issues related to the treatment of patients with end-stage heart disease.

The concept of an alternative list for older patients seems rational but in turn introduces many theoretical and practical concerns. Use of marginal donor hearts for high-risk recipients seems likely to result in reduced recipient survivals both in the immediate post-transplantation period and in the long term. The actuarial survival for patients after all other cardiac surgical operations is influenced by the age of the patient, and there is no logical reason why cardiac transplantation should be different. There are also factors associated with long-term immunosuppression in older patients that could be expected to adversely influence survival and quality of life.

The number of cardiac donors in this country has remained static over the past 10 years despite intense efforts at public education and the aggressive expansion of acceptable donor criteria. Therefore, some upper age limit must be used to prevent further increases in mortality rates for patients awaiting transplantation. Moreover, the upper age limit used by most programs is merely a guideline for selection. Age alone rarely disqualifies patients from consideration. Chronologic age certainly does not strictly correlate with physiologic age in every case. The challenge for transplant physicians is to establish objective parameters that accurately predict the recipient's response to the trauma of the operation and the need for lifelong immunosuppression.

The development of more specific immunosuppressive drugs and the establishment of effective immune tolerance protocols have the potential of reducing the complications associated with immunosuppression that seem to be more frequent and severe for elderly patients. The future implementation of immunosuppressive strategies based on pharmacogenomics may permit the customization of drug therapy guided by the patients' genetic profiles and would be particularly beneficial for elderly patients.

Society must balance the issue of reducing death on the waiting list with survival outcome after transplantation. The expansion of the donor supply with the use of marginal hearts could reduce the chance of death for patients awaiting transplantation. If the results of marginal donor hearts used for transplantation in patients on alternative lists are comparable with the current results with nonmarginal donor hearts, then those hearts should be used for the lower-risk patients who are currently dying on the regular waiting list. The reality is that for the foreseeable future the 
demand for hearts will exceed the supply from the current donor pool even with the liberal use of marginal, high-risk donor hearts.

Xenotransplantation, permanent mechanical support, improved medical therapy, biventricular pacing, cellular transplantation, and gene therapy are potential solutions to the current donor heart shortage. Presently, permanent mechanical support would seem to be the most feasible treatment strategy for patients with end-stage heart disease refractory to medical therapy. The results of prospective trials such as the Randomized Evaluation of Mechanical Assistance Therapy for Congestive Heart Failure (REMATCH) study may provide the necessary guidance for the use of this therapy for these patients.

The objective of balancing the death of patients awaiting transplantation with maximizing post-transplantation survival may be partially achieved by matching the risk of marginal donor heart dysfunction with the immunopathophysiologic function of the high-risk recipients. The United Network for Organ Sharing (UNOS) has made a policy decision regarding the use of pediatric-age donor hearts for pediatric-age recipients. This policy sets a precedent for the matching of the risks donor heart function with recipient parameters. All transplant physicians trying to decide whether to use a specific donor heart for a potential recipient use this concept on a daily basis. All of the donor data and recipient conditions are analyzed in aggregate, and a decision is rendered concerning proceeding with transplantation. The consideration of all donor hearts including the marginal hearts for one list of recipients seems the most reasonable paradigm. The creation of an alternate list seems to disadvantage the patients on this list by giving them second-rate hearts; conversely, if the hearts are acceptable, then the standard list patients are disadvantaged by not being given the chance for the hearts.

The definition of a "marginal" donor heart is frequently in the eye of the beholder. Some of the donor factors associated with this designation include female sex, age over 45 years, dopamine support of greater than $10 \mu \mathrm{g} \times$ $\mathrm{kg}^{-1} \times \min ^{-1}$, body surface area of less than $70 \%$ of recipient body surface area, history of drug abuse, diabetes mellitus, cardiac arrest, anticipated graft ischemic time of more than 210 minutes, central venous pressure of greater than $10 \mathrm{~mm} \mathrm{Hg}$, left ventricular hypertrophy, ventricular dysfunction, risk factors for or the presence of coronary artery disease, and hepatitis. Recipient factors associated with increased risk of post-transplantation mortality include age over 50 years, pulmonary vascular resistance of more than 2.5 Wood units, male sex with a body surface area over $2.5 \mathrm{~m}^{2}$, more than one previous sternotomy, obesity, diabetes mellitus, peripheral vascular disease, diverticulosis, and a history of malignancy. The challenge to match donor and recipient risks will continue until more definitive data are available to guide the decision about which heart should be given to which recipient. On the basis of these observations, I would continue to advocate responsible stewardship of the limited donor resources and select the best candidates for transplantation irrespective of patient age. The concept of establishing an alternative list should not be widely adopted unless more convincing data are provided to support this complex paradigm. 